\title{
Compact high-resolution spectral phase shaper
}

\author{
S. Postma, ${ }^{\text {a) }}$ P. van der Walle, ${ }^{\text {b) }}$ H. L. Offerhaus, and N. F. van Hulst ${ }^{\text {c) }}$ \\ Applied Optics Group, Department of Science and Technology, MESA ${ }^{+}$Institute for Nanotechnology, \\ University of Twente, Enschede, The Netherlands
}

(Received 9 August 2005; accepted 30 October 2005; published online 14 December 2005)

\begin{abstract}
The design and operation of a high-resolution spectral phase shaper with a footprint of only $7 \times 10 \mathrm{~cm}^{2}$ is presented. The liquid-crystal modulator has 4096 elements. More than 600 independent degrees of freedom can be positioned with a relative accuracy of 1 pixel. The spectral shaping of pulses from a broadband Ti:sapphire laser is verified by a hybrid cross-frequency-resolved optical gating/Grenouille measurement and intensity autocorrelation. We demonstrate the ability to split one pulse into two or more pulses with a programmable delay of more than $8.5 \mathrm{ps}$. To our knowledge this represents the most compact high resolution device in liquid-crystal modulator-based shaping to this date. () 2005 American Institute of Physics.
\end{abstract}

[DOI: $10.1063 / 1.2140465$ ]

\section{INTRODUCTION}

Shaping femtosecond pulses is an active field of research. Applications include coherent control of energy transfer in molecules, ${ }^{1}$ selective Raman spectroscopy and microscopy, ${ }^{2,3}$ separation and detection of isotopes and molecules, ${ }^{4}$ probing of femtosecond structural dynamics of macromolecules ${ }^{5,6}$ and coherent laser control of physicochemical processes. ${ }^{7-10}$ Recently, methods based on holographic patterns ${ }^{11}$ have been investigated as well as modulator based shapers with electro-optic, ${ }^{12}$ acousto-optic, ${ }^{13}$ or liquid-crystal spatial light modulators (SLM). ${ }^{14-16}$

In the latter method the modulator is placed in the Fourier plane of the dispersed spectrum. ${ }^{17}$ Most of the modulators currently in use are quite large $(\geqslant 5 \mathrm{~cm}$ for 640 separate electrodes, so that the entire shaping setup requires more than $2000 \mathrm{~cm}^{2}$ ) even for a folded arrangement.

Here, we report an unfolded highly compact setup $\left(7 \times 10 \quad \mathrm{~cm}^{2}\right)$ containing a small high-resolution onedimensional liquid-crystal modulator (LCM) (4096 elements in $7.4 \mathrm{~mm}$ ), that was originally designed for beam steering applications. This modulator can steer beams with maximum deflection angles of $25^{\circ}$, depending on the liquid-crystal birefringence. ${ }^{18}$

The shaping setup is compressed to the point where the spot size for each frequency is comparable to the size of the effective addressable elements ( $\sim 7$ pixels). As a demonstration we show the ability to split one pulse into two or more pulses with a programmable time delay.

\section{SETUP}

The setup consists of a pulsed laser system, the spectral phase shaper and a cross-frequency-resolved optical gating (FROG) setup to analyze the shaped pulses.

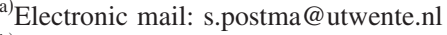

${ }^{b)}$ Present address: FOM Institute for Atomic and Molecular Physics (AMOLF), Amsterdam, The Netherlands.

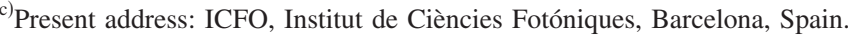

\section{A. Pulsed laser system}

A pulsed Ti:sapphire oscillator (KM Labs), with prismpair dispersion compensation generates $1.9 \mathrm{~nJ}$ pulses at a repetition rate of $80 \mathrm{MHz}$. The Ti:sapphire oscillator is pumped by a Millennia 2000 (Spectra Physics) laser. The intensity autocorrelation of the pulses is measured using a home-built scanning autocorrelator with a 2 photon lightemitting diode (LED) (Osram, LY 5436-TO). The FWHM pulse length at the LED is $25 \mathrm{fs}$ after external prism pair compression (assuming Gaussian shape). The spectrum of the laser pulses is measured with an Ocean Optics HR2000 spectrum analyzer. The FWHM of the spectrum is $70 \mathrm{~nm}$, centered around $790 \mathrm{~nm}$.

\section{B. Spectral phase shaper}

Figure 1 shows the setup of the spectral phase shaper. The shaping element (C) is the 4096 electrode LCM, commercially available as a beam steering device (Boulder Nonlinear Systems). In this device a uniformly thin layer of liquid crystal is sandwiched between two parallel substrates. The front side is coated with a transparent electrode. The reverse side is patterned with closely spaced electrodes (6 $\mathrm{mm}$ by $1 \mu \mathrm{m}$, with a $1.8 \mu \mathrm{m}$ pitch) covered with a highly reflective dielectric coating. The damage threshold is specified at $5 \mathrm{~W} / \mathrm{cm}^{2}$. The LCM is controlled by an interface card, that can be programmed using LABVIEW or $\mathrm{C}++$. The switching time is measured to be $25 \mathrm{~ms}$. This time is mainly limited by the settling time of the liquid-crystal molecules themselves. In our shaper the distance between the front and back electrode is $6.9 \mu \mathrm{m}$ so that the fields effectively spread out to fill the gap between the pixels. ${ }^{19}$ This yields a higher diffraction efficiency but a decrease in resolution. The spread of the field over multiple pixels also reduces the effects of pixellation and the effects of variation between pixels, compared to conventional LCM phase shapers. ${ }^{14}$

The number of degrees of freedom is measured by deflection of monochromatic light. At the maximum first order angle, the number of degrees of freedom is twice the length 

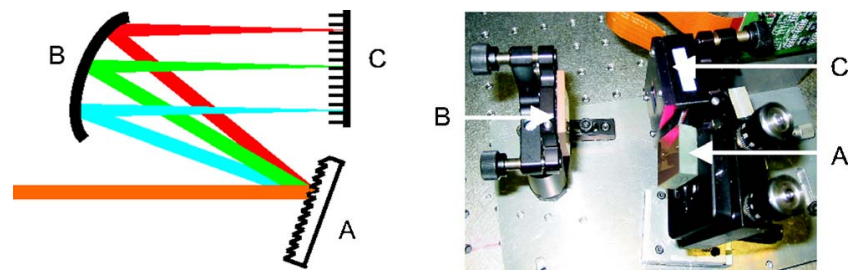

FIG. 1. Schematic and photograph of the spectral phase shaper. (A) grating, (B) cylindrical mirror, and (C) liquid-crystal modulator.

of the active area of the LCM multiplied by the retrieved grating constant. For our shaper this yields approximately 600 degrees of freedom over the complete array $(\approx 7$ pixels per feature). The relative positioning of a spectral feature is still possible at the original resolution of 4096 since each element can be addressed individually. Wang et al. ${ }^{14}$ have demonstrated the influence of nonuniform dispersion on the effective resolution. For our shaper these effect can be compensated using the 4096 elements for the phase profiles and the fact that the electric fields are averaged. Under these conditions the phase shaper can generate pulses with a separation of up to $8 \mathrm{ps}$, limited by the number of degrees of freedom.

To fully exploit the available degrees of freedom of the LCM, the separate "colors" of the laser light must be focused tightly. We expand the beam to an elliptic profile with FWHM diameters of $1.7 \mathrm{~mm}$ (vertically) and $6.4 \mathrm{~mm}$ (horizontally). The beam is dispersed on grating (A) with 830 lines/mm (blazed for $800 \mathrm{~nm}$ with an efficiency of $80 \%$ in the minus-first order) and focused onto the LCM (C) with a cylindrical mirror (B) of a focal distance of $51.7 \mathrm{~mm}$. The monochromatic $1 / e^{2}$ horizontal spotsize is $\sim 5 \mu \mathrm{m}$. It is possible to spread $150 \mathrm{~nm}$ of the spectrum over the active region of the LCM (800 nm in the center). Mapping of the frequency of the incoming light to pixel number is done by identification of edge effects. The amount of birefringence generated by the LCM is measured for five different wavelengths $(760,780,800,840$, and $860 \mathrm{~nm})$.

\section{Advanced joined optimized long-pulse observation technique (AJOLOTE) setup}

To characterize the shaped pulses a hybrid cross-FROG/ Grenouille technique is used. ${ }^{20,21}$ We use a delay line instead of a biprism to extend the delay range, but combine the frequency doubling and wavelength separation in a relatively thick crystal as in a Grenouille setup. In the remainder of the text the device will be referred to as AJOLOTE. Figure 2 shows the setup of the AJOLOTE.

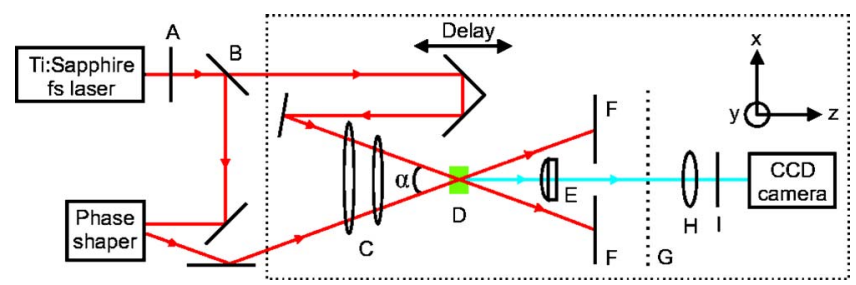

FIG. 2. AJOLOTE setup. (A) half wave plate, (B) pellicle beam splitter, (C) two cylindrical mirrors, (D) $1 \mathrm{~mm}$ thick $\mathrm{BiB}_{3} \mathrm{O}_{6}$ crystal (BIBO), (E) two cylindrical lenses, (F) beam dump, $(\mathrm{G})$ image plane, $(\mathrm{H})$ lens, and (I) filters.
The polarization of the laser pulses is rotated by a half wave plate (A), to suit the $\mathrm{LCM}$ and the $\mathrm{BiB}_{3} \mathrm{O}_{6}$ (BIBO) crystal (D). The pulses are split with a pellicle beam splitter (B). The reference beam passes a programmable delay line, which generates the path length differences between the two beams. The other beam is sent via the phase shaper to the AJOLOTE. Both beams are focused in the $y$ direction by two cylindrical mirrors $(\mathrm{C})$. The BIBO crystal is placed in the center of the focus. BIBO is similar to $\beta$-bariumborate but has a higher nonlinear coefficient $\left[d_{\mathrm{eff}}=3.2 \mathrm{pm} / \mathrm{V}\right.$ (Ref. 22) $]$ and higher dispersion. ${ }^{23}$ The $1 \mathrm{~mm}$ thickness of the crystal meets the conditions described by O'Shea et al. ${ }^{24}$ The BIBO crystal is cut at $28.9^{\circ}$ in the $y z$ plane for type I $(e+e \rightarrow o)$ second harmonic generation. The aperture of the BIBO crystal is $2.8 \mathrm{~mm}$ ( $x$ direction $) \times 3.0 \mathrm{~mm}$ ( $y$ direction). The diameter of the beams is reduced to $1.2 \mathrm{~mm}$ (FWHM), to prevent diffraction effects from the sides of the BIBO crystal.

The full angle $(\alpha)$ between the two beams in the $x$ direction is $35 \mathrm{mrad}$. The divergence in the $x$ direction after the two focusing mirrors is $90 \mathrm{mrad}$ resulting in an internal divergence of $50 \mathrm{mrad}$. These parameters result in a $1.7 \mathrm{~nm}$ (FWHM) phase-matching bandwidth for a fixed angle and an operating range from 712 to $973 \mathrm{~nm}$. The lens (E) consists of two uncoated cylindrical lenses, glued together with optical adhesive (NOA60). One cylindrical lens collimates the different phase matched angles (frequency axis) along the $y$ axis $(f=50 \mathrm{~mm})$ of the image plane $(\mathrm{G})$. The second cylindrical lens images the $x$ axis location where the doubled light is generated $(f=100 \mathrm{~mm})$ on the $x$ axis of the image plane (G). The $x$ axis contains some time delay information for a limited time span that corresponds to the crossing angle. The incoming beams are blocked (F) and the image of the center beam on the image plane is projected with an uncoated $35 \mathrm{~mm}$ lens $(\mathrm{H})$ onto the camera. One line on the $x$ axis is selected and only the programmable delay line is used to generate the time axis of the AJOLOTE image. Two Schott BG 39 filters (I) are placed in front of the charged coupled device camera (monochrome, Pixellink PL-A741) to minimize the fundamental light. The measured patterns on the camera are corrected for the calculated frequency dependence of Fresnel refections, measured filter transmissions and the specified frequency dependence of the camera sensitivity.

\section{RESULTS}

To demonstrate the performance of the phase shaper, a binary phase function is superimposed, that generates a double pulse. The function is a single period square modulation of the spectrum that is corrected for the nonlinearity of the wavelength versus pixel and the nonlinear birefringence of the liquid crystal. Figure 3(a) shows a spectral phase function to generate two pulses with a delay of 980 fs. Figure 3(b) shows the calculated pulse shape in the time domain. For this phase function there are side lobes at multiples of $980 \mathrm{fs}$. The FWHM of the two pulses is $15 \mathrm{fs}$, which is the Fourier limit of the applied spectrum.

Figure 4 shows a measurement of the shaped pulse with the AJOLOTE. The image in Fig. 4(a) shows the delay time 


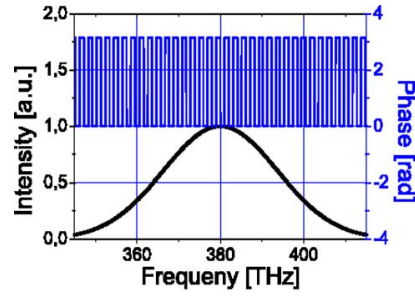

(a)

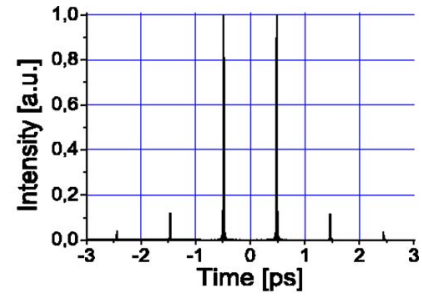

(b)
FIG. 3. (a) Applied spectral phase function with spectral intensity to create a double pulse as shown in the calculated temporal intensity profile (b).

between the two pulses. The image is reconstructed using an iterative algorithm ${ }^{25}$ in Fig. 4(b). For this reconstructing negative chirp is added to the reference pulse, because the prism compressor is also used to compensate the dispersion added in the $2 \mathrm{~mm}$ cover glass from the LCM. The reconstructed spectral intensity with spectral phase are shown in Fig. 4(c). The corresponding reconstructed time profile with phase is shown in Fig. 4(d). The residual difference between the two images equals $8.3 \times 10^{-3}$, calculated as defined in Ref. 26.

The spectral phase function of Fig. 3(a) is not fully retrieved in the shape of the phase function in Fig. 4(c). The algorithm has put higher order modulation in the amplitude of the spectrum, due to the restricted time window, which cuts pulses at larger delays. The retrieved temporal intensity faithfully recovers the delay time of 980 fs.

Figure 5 shows the delay between the maxima of the pulses versus the number of periods on the phase shaper. A linear fit through the experimental data yields a slope of $28.1 \mathrm{fs} /$ period. Which is in excellent agreement with the calculated slope.

\section{CONCLUSIONS}

We have developed and realized a very compact (7 $\times 10 \mathrm{~cm}^{2}$ ) spectral phase shaper device. The shaped pulses

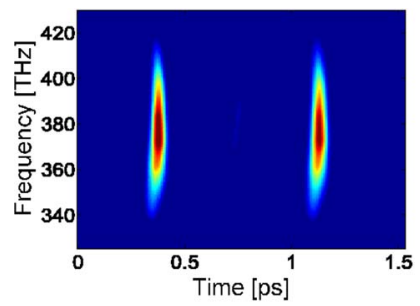

(a)

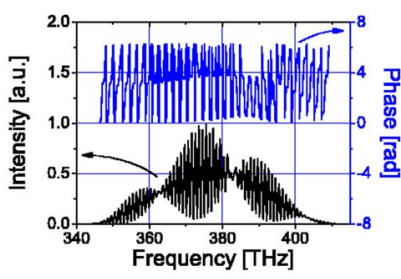

(c)

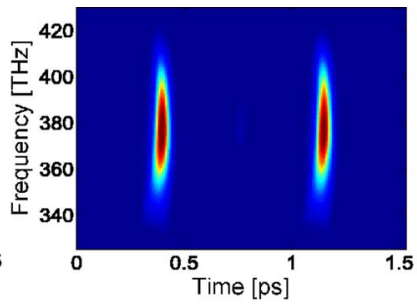

(b)

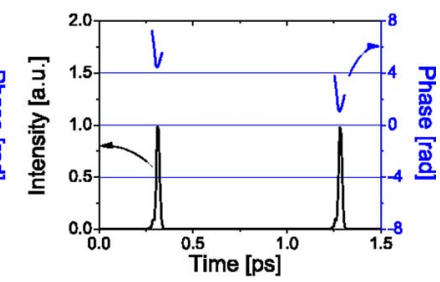

(d)
FIG. 4. (a) Measured AJOLOTE-image, (b) retrieved AJOLOTE-image, (c) reconstructed spectral intensity and spectral phase, and (d) reconstructed temporal intensity and temporal phase.

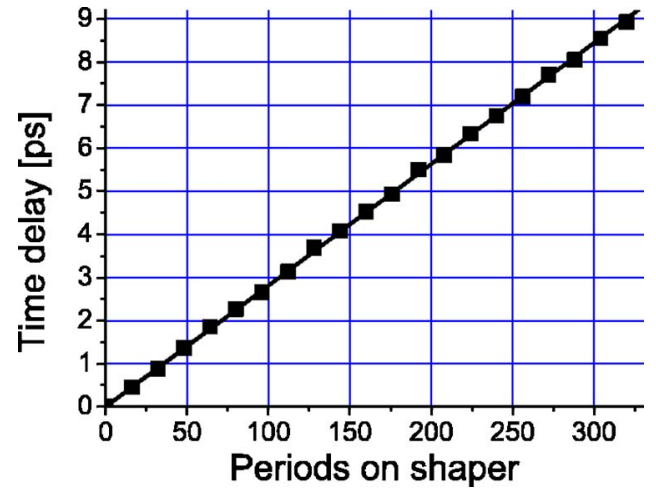

FIG. 5. Number of periods on the LCD versus the delay time between two pulses originating from one single pulse. Experimental ( $\square$ ), and calculated (-) data.

are characterized with an AJOLOTE setup. The double pulse and the controllable delay of more than 8.5 ps confirms the operation and spectral resolution of the shaper beyond the conservative estimate of 8 ps.

This device offers an improved stability compared to a Michelson interferometer for generating double pulses. Because the two pulses follow the same path, unwanted path length variations are eliminated. The small dimensions of the shaper assure extra robustness. The phase shaper setup is flexible, as computer control allows for rapidly changing pulse shapes. Applications include selective two photon microscopy and spectroscopy with the same setup, using only different pulse shapes.

We intend to apply these pulses to reduce the problems caused by premature photograph bleaching in single molecule pump-probe experiments, ${ }^{6}$ where the phase shaper allows rapid and flexible changing of the delay times.

\section{ACKNOWLEDGMENTS}

The authors are grateful to W. van der Vecht for useful suggestions. This work is part of a research project of the "Stichting voor Fundamenteel Onderzoek der Materie (FOM)," which is financially supported by the "Nederlandse Organisatie voor Wetenschappelijk Onderzoek (NWO)."

${ }^{1}$ J. L. Herek, W. Wohlleben, R. J. Cogdell, D. Zeidler, and M. Motzkus, Nature (London) 417, 533 (2002).

${ }^{2}$ I. Pastirk, J. M. Dela Cruz, K. A. Walowicz, V. V. Lozovoy, and M. Dantus, Opt. Express 11, 1695 (2003).

${ }^{3}$ M. Dantus and V. V. Lozovoy, Chem. Rev. (Washington, D.C.) 104, 1813 (2004).

${ }^{4}$ F. Vetter, M. Plewicki, A. Lindinger, A. Merli, S. M. Weber, and L. Woste, Phys. Chem. Chem. Phys. 7, 1151 (2005).

${ }^{5}$ P. F. Tian, D. Keusters, Y. Suzaki, and W. S. Warren, Science 300, 1553 (2003).

${ }^{6}$ E. M. H. P. van Dijk, J. Hernando, J. J. Garcia-Lopez, M. Crego-Calama, D. N. Reinhoudt, L. Kuipers, M. F. Garcia-Parajo, and N. F. van Hulst, Phys. Rev. Lett. 94, 078302 (2005).

${ }^{7}$ D. Meshulach and Y. Silberberg, Nature (London) 396, 239 (1998).

${ }^{8}$ T. Brixner, N. H. Damrauer, P. Niklaus, and G. Gerber, Nature (London) 414, 57 (2001).

${ }^{9}$ H. Rabitz, R. de Vivie-Riedle, M. Motzkus, and K. Kompa, Science 288 , 824 (2000).

${ }^{10}$ Y. Silberberg, Nature (London) 430, 624 (2004).

${ }^{11}$ Y. Ding, R. M. Brubaker, D. D. Nolte, M. R. Melloch, and A. M. Weiner, Opt. Lett. 22, 718 (1997).

${ }^{12}$ M. Haner and W. S. Warren, Appl. Phys. Lett. 52, 1458 (1988). 
${ }^{13}$ C. W. Hillegas, J. X. Tull, D. Goswami, D. Strickland, and W. S. Warren, Opt. Lett. 19, 737 (1994).

${ }^{14}$ H. Wang, Z. Zheng, D. E. Leaird, A. M. Weiner, T. A. Dorschner, J. J. Fijol, L. J. Friedman, H. Q. Nguyen, and L. A. Palmaccio, IEEE J. Quantum Electron. 7, 718 (2001).

${ }^{15}$ A. M. Weiner, Rev. Sci. Instrum. 71, 1929 (2000).

${ }^{16}$ A. Präkelt, M. Wollenhaupt, A. Assion, Ch. Horn, C. Sarpe-Tudoran, M. Winter, and C. Baumert, Rev. Sci. Instrum. 74, 4950 (2003).

${ }^{17}$ D. Goswami, Phys. Rep. 374, 385 (2003).

${ }^{18}$ B. Apter, E. Bahat-Treidel, and U. Efron, Opt. Eng. (Bellingham) 44, 054001 (2005).

${ }^{19}$ J. Stockley and S. Serati, Proc. SPIE 5550, 32 (2004).
${ }^{20}$ R. Trebino, Frequency-Resolved Optical Gating: The Measurement of Ultrashort Laser pulses (Kluwer Academic, Dordrecht, The Netherlands, 2000).

${ }^{21}$ P. O'Shea, S. Akturk, M. Kimmel, and R. Trebino, Appl. Phys. B 79, 683 (2004).

${ }^{22}$ M. Ghotbi and M. Ebrahim-Zadeh, Opt. Express 12, 6002 (2004).

${ }^{23}$ H. Hellwig, J. Liebertz, and L. Bohaty, J. Appl. Phys. 88, 240 (2000).

${ }^{24}$ P. O'Shea, M. Kimmel, and R. Trebino, J. Opt. B: Quantum Semiclassical Opt. 4, 44 (2002).

${ }^{25}$ D. J. Kane, IEEE J. Quantum Electron. 4, 278 (1998).

${ }^{26}$ K. W. Delong and R. Trebino, J. Opt. Soc. Am. B 11, 2429 (1994). 